\title{
Sky coverage modeling for the whole sky for laser guide star multiconjugate adaptive optics
}

\author{
Lianqi Wang, ${ }^{1, *}$ David Andersen, ${ }^{2}$ and Brent Ellerbroek ${ }^{1}$ \\ ${ }^{1}$ Thirty Meter Telescope Project, 1111 South Arroyo Parkway, Suite 200, Pasadena, California 91105, USA \\ ${ }^{2}$ Herzberg Institute of Astrophysics, 5071 W. Saanich Road, Victoria, British Columbia V9E 2E7, Canada \\ ${ }^{*}$ Corresponding author: lianqiw@tmt.org
}

Received 17 February 2012; revised 23 March 2012; accepted 26 March 2012; posted 28 March 2012 (Doc. ID 163237); published 1 June 2012

\begin{abstract}
The scientific productivity of laser guide star adaptive optics systems strongly depends on the sky coverage, which describes the probability of finding natural guide stars for the tip/tilt wavefront sensor(s) to achieve a certain performance. Knowledge of the sky coverage is also important for astronomers planning their observations. In this paper, we present an efficient method to compute the sky coverage for the laser guide star multiconjugate adaptive optics system, the Narrow Field Infrared Adaptive Optics System (NFIRAOS), being designed for the Thirty Meter Telescope project. We show that NFIRAOS can achieve more than $70 \%$ sky coverage over most of the accessible sky with the requirement of $191 \mathrm{~nm}$ total $\mathrm{rms}$ wavefront. (C) 2012 Optical Society of America
\end{abstract}

OCIS codes: $\quad 010.1080,010.7350$.

\section{Introduction}

Adaptive optics (AO) systems have been developed for ground-based astronomical telescopes to compensate for atmospheric turbulence effects. However, because of the limited availability of bright natural stars, laser guide stars (LGS; based on Rayleigh backscatter [1] or resonance fluorescence from mesosphere sodium atoms) are increasingly used by current $[\underline{2}-4]$ and future $[\underline{5}, \underline{6}]$ ground-based astronomical telescopes. However, because the laser beacons are projected from the ground, the corresponding wavefront sensor (WFS) cannot measure tip/tilt reliably. Therefore, low order natural guide star (NGS) wavefront sensors are generally necessary to provide tip/tilt measurement. For AO systems with modest to large fields of view, such as multiconjugate AO (MCAO) or multiobject AO concepts, several natural guide stars are also necessary to provide the field dependent tip/tilt information, or plate scale modes. Besides, in the case of sodium laser guide star,

1559-128X/12/163692-09\$15.00/0

(C) 2012 Optical Society of America because of the evolution of sodium atom densities, the focus measurement has to be corrected by focus information from low order $2 \times 2$ NGS measurements.

Sky coverage describes the probability of finding NGS within the allowable patrol field that are bright enough to achieve a certain performance. For modest to wide field AO systems, past research $[7,8]$ has found that three low order NGS are generally necessary to accurately sense the global tip/tilt and tilt anisoplanatism modes, without unacceptably limiting the sky coverage. At times, two or even one low order NGS is enough to sense these modes if they are located in the right place (i.e., close enough to the science target). For an extremely large telescope, sensing in infrared band ( $J$ and/or $H$ ) and tracking the diffraction-limited point spread function (PSF) cores is also necessary to achieve milli-arcsecond level residual tip/tilt error.

Evaluating the sky coverage of such systems with high fidelity is difficult because of the high dimensional nature (eight dimensions to be exact, which are magnitude and coordinate of three stars minus overall rotation) of finding three stars and evaluating the performance. Previously the sky coverage had 
been evaluated with approximate models that only take into account the average Strehl of NGS at a given location [9-11] before we, at the Thirty Meter Telescope project (TMT), developed a high fidelity sky coverage simulator based upon a postprocessing technique enabled by the split control of LGS and NGS modes [12]. This method has recently been proven to give reliable performance estimates compared to integrated simulations [13].

So far, to our best knowledge, the sky coverage for LGS MCAO systems has been evaluated only for a given telescope zenith angle and certain star statistics (at the galactic pole, or 30 degrees galactic latitude) [9-11]. However, knowing the fractional sky coverage at one point in the sky does not give astronomers a sense of how likely it is to be able to observe targets at any given point on sky. Gemini had a sky coverage map for the classic LGS AO system [14], which is simply a star availability map. For MCAO systems, not only the brightness of the stars but also their location in the field of view (FoV) determine the performance. In this paper, we present the sky coverage maps for the Narrow Field Infrared Adaptive Optics System (NFIRAOS) on TMT as a function of galactic latitude and longitude for the Mauna Kea site. With our tools, it is also straightforward to study the sky coverage of specific fields, like the Hubble ultradeep field [15] or to select stars in any field where existing IR stars of down to magnitude 22 are known.

The following sections are organized as follows. Section 2 presents the parameters of the TMT AO system NFIRAOS, which our modeling studies. Section 3 reviews our methods to calculate sky coverage. Section 4 describes our modeling for star counts and zenith angle as a function of galactic coordinates and hour angle. Section 5 presents the results of the study, and Section $\underline{6}$ gives the conclusion.

\section{NFIRAOS}

The TMT NFIRAOS [16] is an MCAO system with two deformable mirrors (DMs) conjugated to ranges of 0 and $11.2 \mathrm{~km}$. An asterism of five sodium LGS arranged in a pentagon of 35 arc-second radius plus sixth on axis are sensed by LGS WFS. Up to three NGS (if available) are picked off by low order oninstrument wavefront sensors.

The performance requirements for NFIRAOS include diffraction-limited turbulence compensation from $0.8 \mu \mathrm{m}$ to $2.5 \mu \mathrm{m}$ over fields of view of up to 30 arc-second in diameter. In particular, the rms image jitter should be less than 2 mas for median sky coverage at the north galactic pole with median seeing conditions. The NGS are acquired via probe arms within a 2 arcminute diameter circular patrol field of view. The NGS WFS will operate in the near infrared (NIR) ( $J$ and $H$ band) to take advantage of partial sharpening of the stars by NFIRAOS. [The K band had been excluded because originally the selected detector (Hawaii-1RG) had negligible quantum efficiency at this wavelength. This may change once we baseline our design on the newly designed Hawaii2RG.] In addition, NIR NGSs are more likely to be available, especially in regions that are obscured by dust. Faint halo stars are also more likely to be red, which increases the number of potential NGSs at a given magnitude. The WFS pixel size will be matched to the Nyquist sampling of the PSF to improve the linearity and signal to noise ratio. Additional system and simulation parameters are summarized in Table $\underline{1}$.

Table 1. NFIRAOS and Atmospheric Simulation Parameters

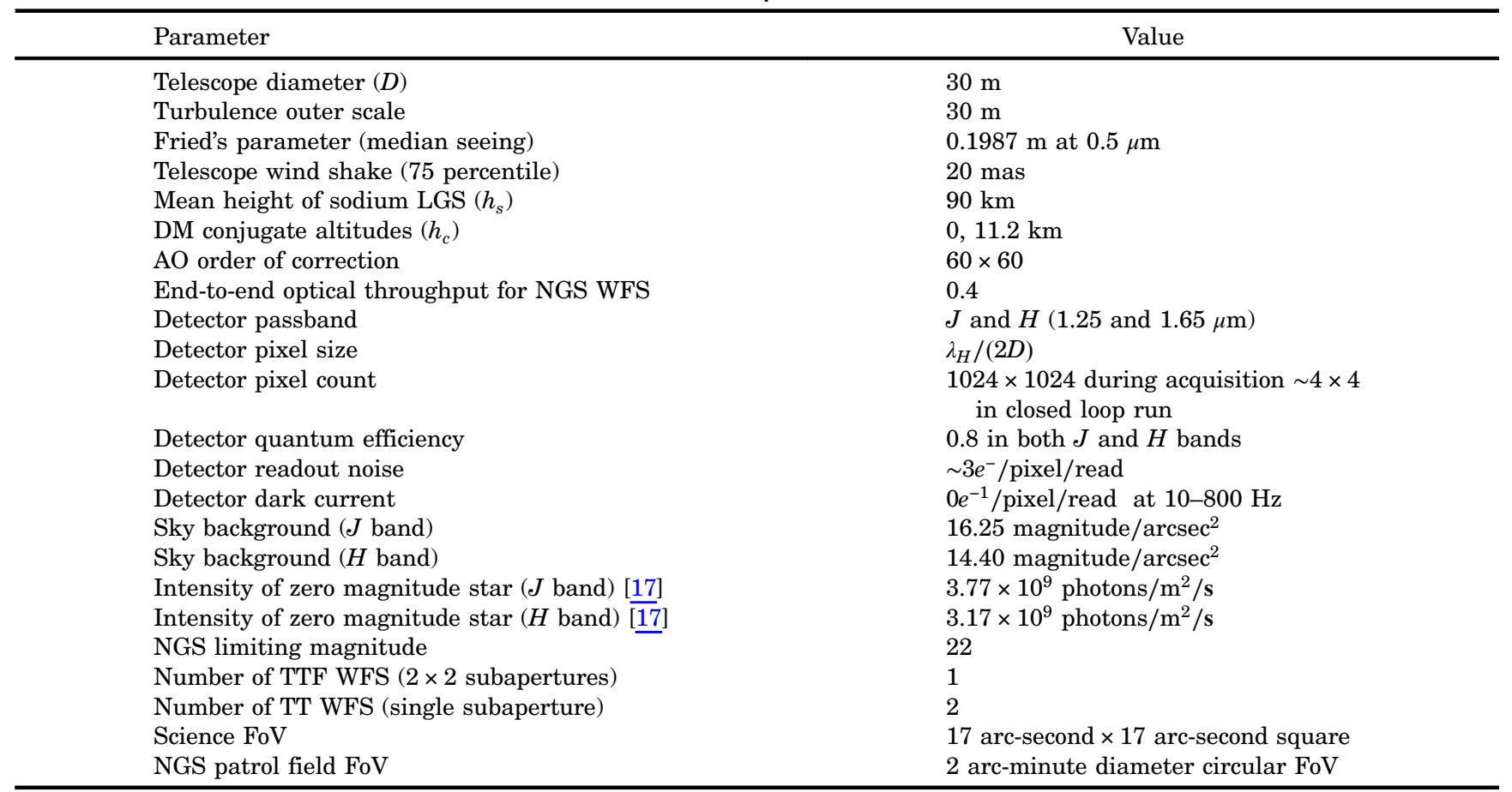


Table 2. $C_{n}^{2}$ Profile Models for the Mauna Kea 13 N Site ${ }^{a}$

\begin{tabular}{ccccc}
\hline & \multicolumn{3}{c}{ Layer weighting } & \\
\cline { 2 - 4 } Altitude $(\mathrm{km})$ & $25 \% r_{0}=0.247 \mathrm{~m}$ & $50 \% r_{0}=0.186 \mathrm{~m}$ & $75 \% r_{0}=0.135 \mathrm{~m}$ & wind speed $(\mathrm{m} / \mathrm{s})$ \\
\hline 0 & 0.5152 & 0.4557 & 0.3952 & 5.6 \\
0.5 & 0.0951 & 0.1295 & 0.1665 & 5.8 \\
1 & 0.0322 & 0.0442 & 0.0703 & 7.3 \\
2 & 0.0262 & 0.0506 & 0.0773 & 13.3 \\
4 & 0.116 & 0.1167 & 0.0995 & 10.1 \\
8 & 0.0737 & 0.0926 & 0.0843 & 12.1 \\
\hline
\end{tabular}

${ }^{a}$ TMT has no direct measurement of the outer scale. We use a conservative value of $30 \mathrm{~m} \mathrm{[18]}$.

The split tomography control algorithm will be used in both sky coverage simulations and the actual real time controller for NFIRAOS. In this control algorithm, the separate LGS and NGS control loops are driven independently. The atmospheric tomography step of the LGS control loop applies a minimum variance estimator [19] to tip/tilt removed, pseudo open loop LGS WFS gradients. The NGS control loop uses a noise-weighted, least squares reconstructor and can operate at a different frame rate than the LGS loop, depending upon the brightness of the NGS. The separation between the LGS and NGS loops is improved by nulling the component of the LGS-controlled DM commands, which lies in the span of the NGS-controlled modes. The details in the split tomography concept can be found in $[\underline{12}, \underline{20]}$.

\section{Sky Coverage}

The high fidelity sky coverage simulations of MCAO systems had been presented in [12]. Here we will give only a brief review. The NFIRAOS split tomography control architecture described in Section 2 enables the efficient modeling and evaluation of the LGS and NGS control loops as a two step process. The first step is a simulation of the high-order LGS control loop using our in-house developed multithreaded AO simulator (MAOS) [21] that can execute each AO time step in just $0.1 \mathrm{~s}$ wall clock time. During this step, we simulate seven layers of atmosphere based on $C_{n}^{2}$ profiles measured at the Mauna Kea $13 \mathrm{~N}$ site [22], as shown in Table 2, at 1/64 meter sampling and use physical optics to model the LGS WFS $[23,24]$. The five NGS-controlled modes (or global tip/tilt and plate scale modes) contained in the atmosphere are corrected perfectly, using least squares fits, to minimize the wavefront error (WFE) over the 17 arc-second square science FoV. Time histories of the resulting NGS WFS PSFs, the best fit of NGS modes, and the associated geometric gradients (Zernike best fit) WFS measurements are recorded for an array of both tip/tilt (TT) and tip/tilt/focus/ astigmatism (TTF) wavefront sensors positioned at 113 NGS locations arranged on a square grid with 10 arc-second spacing across the NFIRAOS 2 arcminute patrol FoV. A total of 5000 time steps are simulated for each seed. The range and strength of turbulence layers, and the range and signal level of the laser guide stars, are scaled by the zenith angle.
During the postprocessing step, the following steps are then carried out:

1. We first generate a random star field according to the star statistics based on the Besançon model $[25,26]$. The number of stars is obtained from a Poisson random number draw with the average number of $J<22$ stars in the patrol FoV. The magnitude is then determined from the distribution function. The location of the stars is uniformly distributed across this FoV.

2. For this star field, we then generate all possible three-star asterisms, with a TTF and two TT WFS (if not enough stars are available, we will fall back to a single TTF or one TTF with one TT WFS).

3. For each asterism, the gradient measurement error attributable to noise is computed based on the averaged PSF (interpolated from the saved PSFs) and star magnitudes [23]. Then the type II controller [27] is optimized, and the residual WFE in the NGS modes is estimated based upon the measurement error power spectral density (PSD) of the NGS mode errors attributable to the combined effects of turbulence and telescope wind shake [13,28]. An additional sodium focus tracking error is estimated from the sodium height PSD and the sampling frequency [29].

4. We then select a few asterisms that give close to minimum WFE and record the corresponding range of NGS sampling frequencies.

5. For each of the selected asterism and sampling frequency combinations, we then evaluate the performance in the time domain using a postprocessing method [12]. For each time step:

(a) We add the residual NGS mode error back into the NGS PSF, sample the PSF onto the WFS detector, and add noise.

(b) We take NGS WFS measurements and apply the NGS reconstructor.

(c) We then apply the correction using a type II controller with gains optimized in step 3.

This step allows physical optics effects and the actual NGS WFS pixel processing algorithm to be factored into the performance estimate. We then estimate the rms WFE after the loop has stabilized.

6. Finally, we select the asterism and sampling frequency that gives the minimum WFE. 
7. We then repeat steps 1 through 6 for a new random star field (500 in total) and finally collect statistics.

The preceding process gives the sky coverage statistics for a given telescope zenith angle and guide star density model. We carried out the process for eight different zenith angles between 0 and $65^{\circ}$ and seven different stellar densities. The results are shown in Section 5 .

\section{Zenith Angle and Stellar Density Maps}

Once the NFIRAOS NGS WFE results have been calculated on a grid of zenith angles and stellar densities, we need to project the results onto astronomical coordinates. We begin the process by creating maps of a zenith angle for a given hour angle and stellar densities.

\section{A. Zenith Angle Map}

The telescope zenith angle is a function of both hour angle and declination. We begin by calculating the declination $(\delta)$ for each galactic latitude $(b)$ and longitude $(l)$ [30]:

$$
\sin (\delta)=\cos (b) \cos \left(l_{\mathrm{CP}}-l\right) \cos \left(\delta_{\mathrm{GP}}\right)+\sin (b) \sin \left(\delta_{\mathrm{GP}}\right),
$$

where $l_{\mathrm{CP}}=123.93^{\circ}$ is the longitude of the north celestial pole and $\delta_{\mathrm{GP}}=27.13^{\circ}$ is the declination of the north galactic pole. The Zenith angle $(z a)$ for the hour angle of $h$ can then be calculated as

$$
\cos (z a)=\sin (\delta) \sin (L)+\cos (\delta) \cos (L) \cos (h),
$$

where $L$ is the latitude of the telescope $\left(19.83^{\circ}\right.$ for Mauna Kea $13 \mathrm{~N}$ ). Figure 1 shows the zenith angle in galactic coordinates for zero hour angle.

\section{B. Stellar Density Map}

To create a sky coverage map for a large part of the sky without running too many simulations, we need to parameterize the distribution of stars as a function of brightness over the whole sky. Luckily, at high galactic latitudes, the normalized cumulative distribution function of stars as a function of $J$ band magnitude are very similar (see Fig. 2). If normalized using star counts at $J \leq 19$ (80\% of the selected TTF

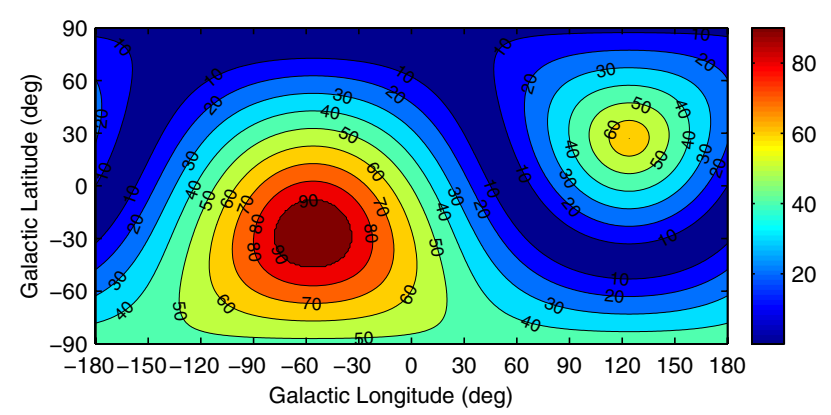

Fig. 1. (Color online) Zenith angle in galactic coordinates for zero hour angle. Zenith angles above $65^{\circ}$ are not reachable by TMT.

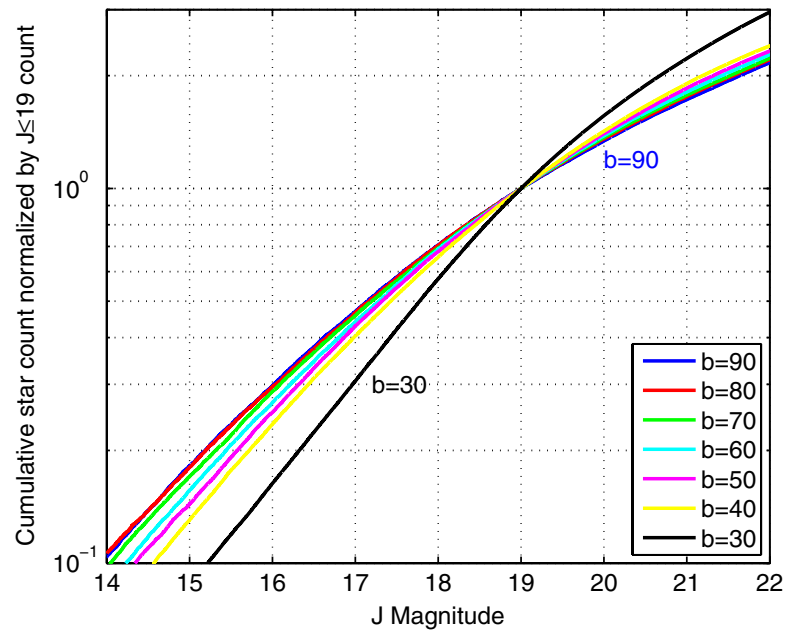

Fig. 2. (Color online) Normalized integrated star counts are very similar at high galactic latitudes but become remarkably different at low galactic latitudes (black line).

stars have $J \leq 19 ; 60 \%$ of the brightest TT star have $J \leq 19$ at the north galactic pole), the cumulative distribution functions differ by less than $10 \%$ where $16 \leq J<22$. We therefore adopt the shape of the cumulative stellar density function for the north galactic pole and run simulations with a few different normalization factors corresponding to the number of stars with $J \leq 19$. At the north galactic pole, there are about 2300 stars $/ \mathrm{deg}^{2}$ with $J \leq 19$.

This cumulative density function model starts to break down for $J \leq 19$ densities of 7500 stars $/ \mathrm{deg}^{2}$, corresponding to about $30^{\circ}$ galactic latitude, because, closer to the galactic plane and bulge, stars change radically owing to contributions from disk and bulge populations (see the curve labeled with $b=30$ in Fig. 2). For these locations, we use the pessimistic assumption that the stellar density plateaus at 7500 stars $/ \mathrm{deg}^{2}$ and the shape of the distribution function is unchanged. In reality, performance for NFIRAOS is already quite acceptable, even with this pessimistic assumption.

To generate the stellar density map, we queried the Besançon model [25] using galactic latitudes in the range between 30 and $90^{\circ}$, and longitudes between 0 and $180^{\circ}$ ), assuming the densities are symmetric with positive and negative latitudes or longitudes. We interpolate these points to finer grids. The resulting map is shown in Fig. $\underline{3}$.

\section{Results}

To obtain the sky coverage map in the galactic coordinate, we carried out sky coverage simulations for a wide range of zenith angles at $0,30,45,55,57.5,60$, 62.5 , and $65^{\circ}$, and for $J \leq 19$ star counts of 2300 (corresponding to galactic pole), $2800,3700,4500,5500$, 6500 , and 7500 stars $/ \mathrm{deg}^{2}$. For each simulation, we averaged the performance over four different turbulence seeds and two different asterism seeds. To sample a range of turbulence conditions, we carried out all the simulations for the $25 \%$, median, and $75 \%$ 


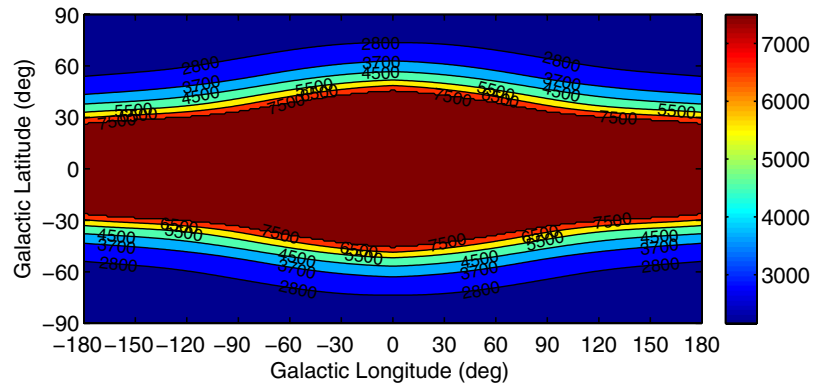

Fig. 3. (Color online) Stellar density map in the galactic coordinates. We limited the maximum value to $7500 \mathrm{stars} / \mathrm{deg}^{2}$.

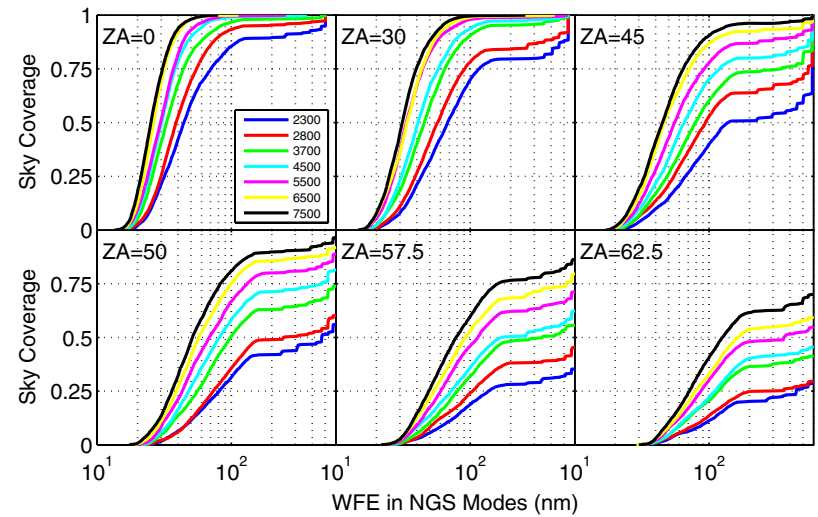

(a) $25 \%$ seeing

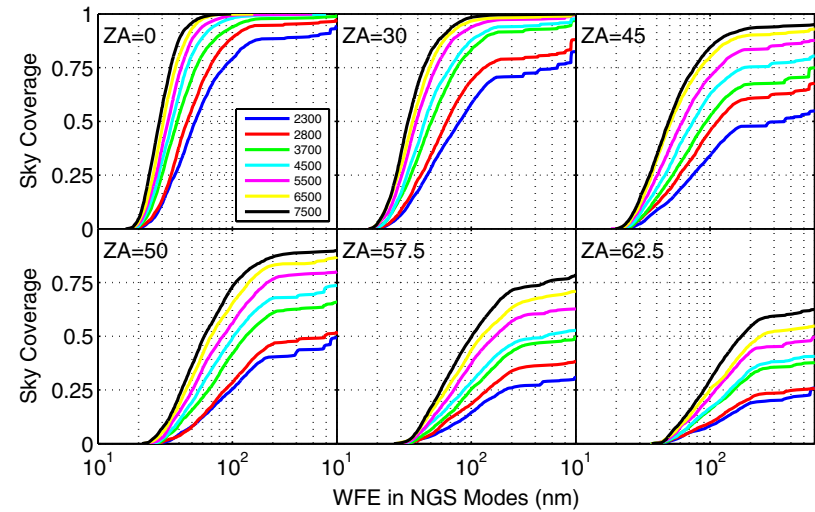

(b) median seeing

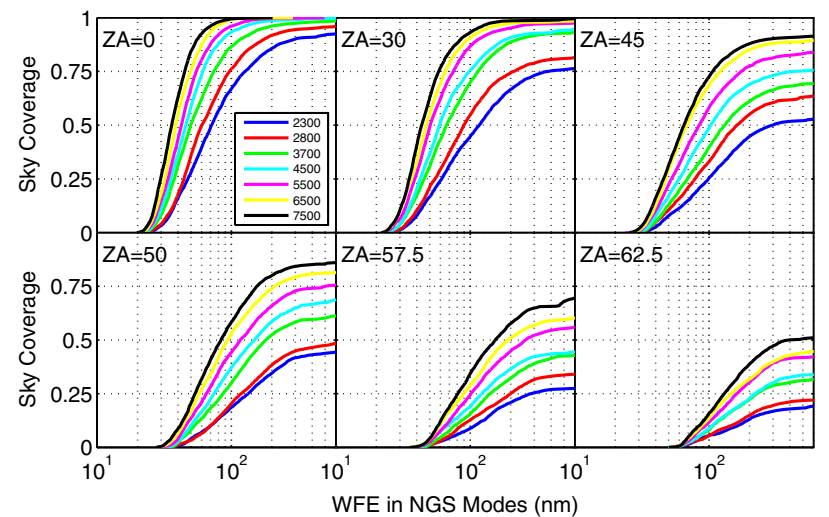

(c) $75 \%$ seeing

Fig. 4. (Color online) Sky coverage (cumulative probability density function) curve for select zenith angles and various $J \leq 19$ star counts for $25 \%$, median, and $75 \%$ seeing conditions. seeing conditions of the Mauna Kea $13 \mathrm{~N}$ site. We used realistic seven layer profiles generated from the TMT site testing survey MK13 N area [22,31]. To assemble representative profiles corresponding to the quartiles of residual WFE, the more than 10000 individual profiles were sorted by uncorrectable residual WFEs (fitting plus server lag error), and $10 \%$ of the profiles clustered around the quartiles were averaged [32] (see Table 2 ).
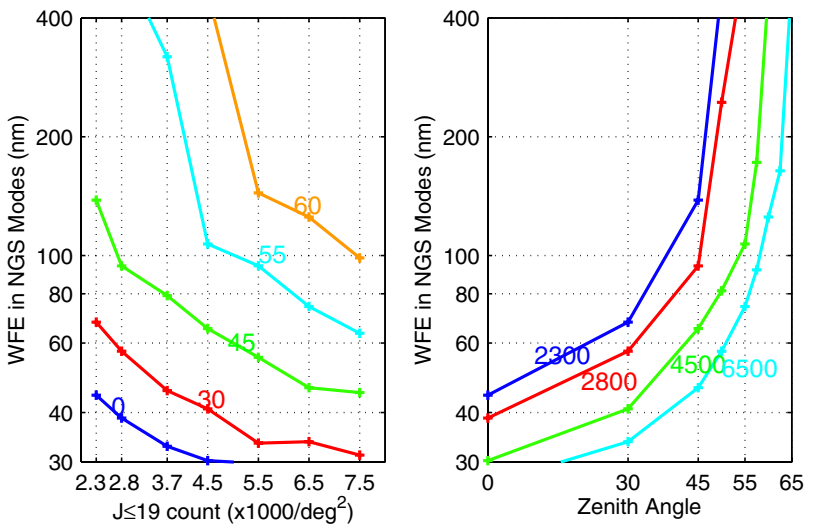

(a) $25 \%$ seeing
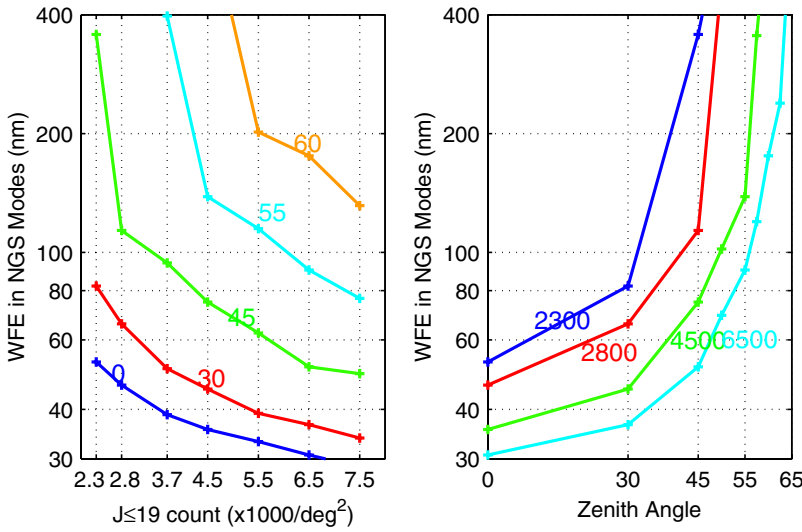

(b) median seeing
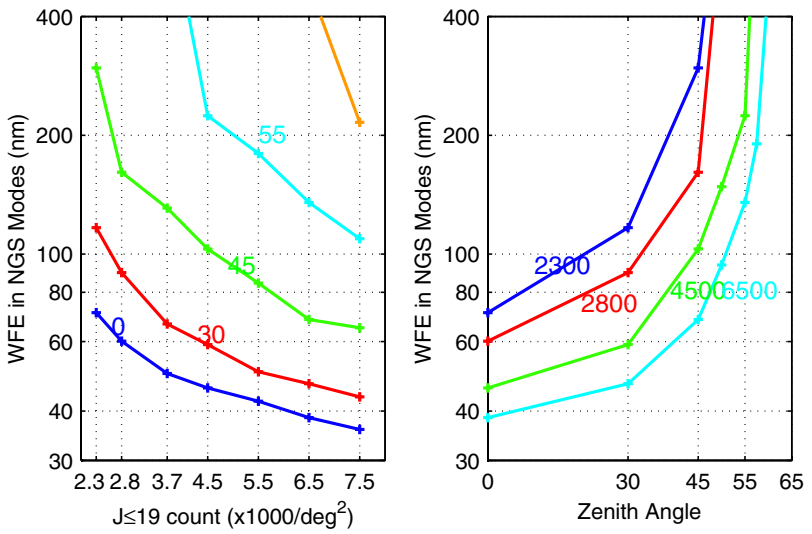

(c) $75 \%$ seeing

Fig. 5. (Color online) Median sky coverage as a function of zenith angle (left panel) or $J \leq 19$ star counts (right panel). The numbers shown along the curves are zenith angle (left panel) and $J \leq 19$ star counts per square degree. 

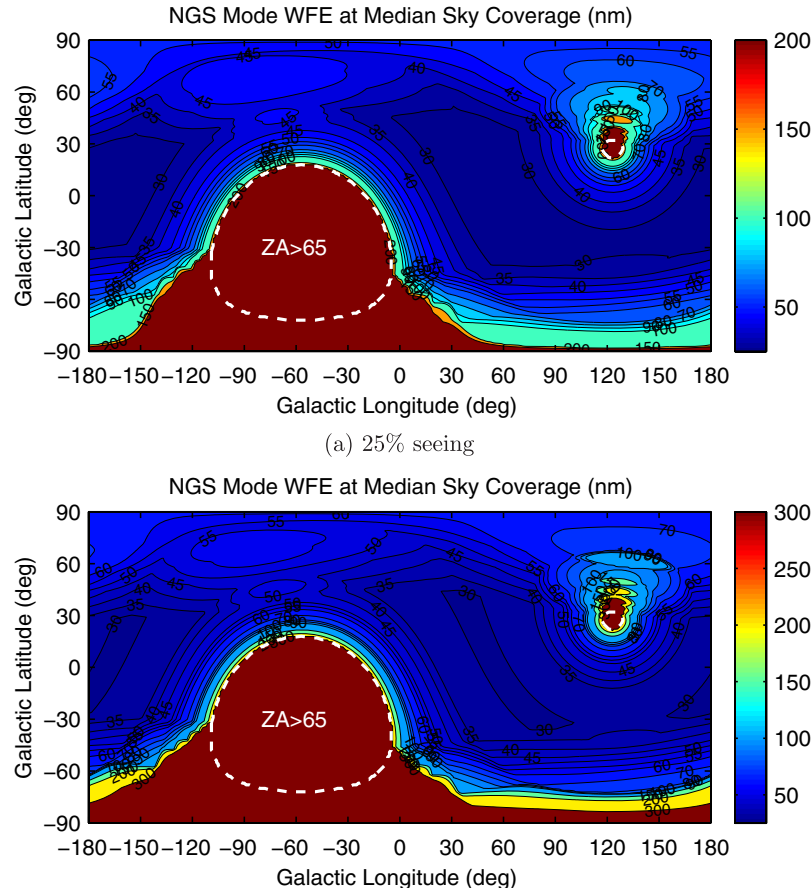

(b) median seeing

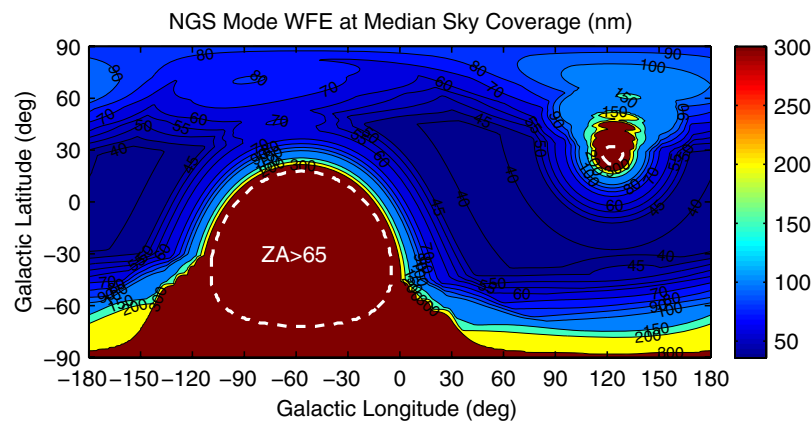

(c) $75 \%$ seeing

Fig. 6. (Color online) Map of rms WFE in NGS-controlled modes at median sky coverage. Hour angle is $0 \mathrm{~h}$. The areas enclosed by dashed white curves are not reachable by TMT owing to zenith angle greater than $65^{\circ}$.

Figure 4 shows the sky coverage (cumulative probability density function) curves for select zenith angles and various $J \leq 19$ star counts for $25 \%$, median, and $75 \%$ seeing conditions. The results are shown as averaged rms WFE in the NGS-controlled modes (tip/tilt plus three plate scale modes) over the 17 arc-second square field of view. It is worth noting that for a $30 \mathrm{~m}$ telescope like TMT, $36 \mathrm{~nm}$ of tip/tilt WFE corresponds to 1 mas tip/tilt jitter. In general, the sky coverage improves with larger stellar densities but degrades with increasing zenith angles or increased turbulence conditions. The degradation is particularly significant for zenith angles greater than $45^{\circ}$ because of the following: (1) reduced sharpening of the NGS PSF, and (2) increased tip/tilt jitter owing to increased turbulence. To gain more insight into the median performance, we also plotted the median sky coverage results as a function of

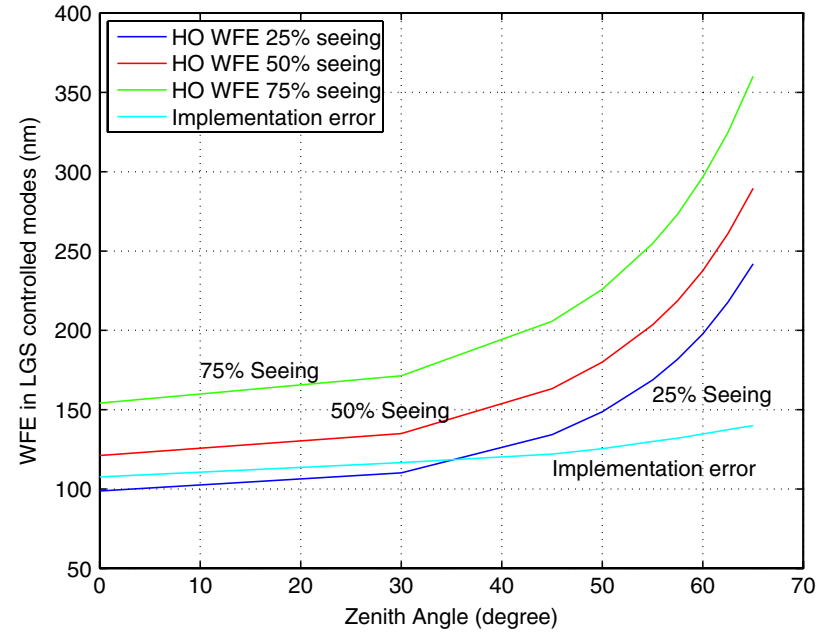

Fig. 7. (Color online) WFEs in LGS-controlled modes (excluding implementation error) and implementation errors, in $\mathrm{nm}$, averaged over the 17 arc-second square field of view.

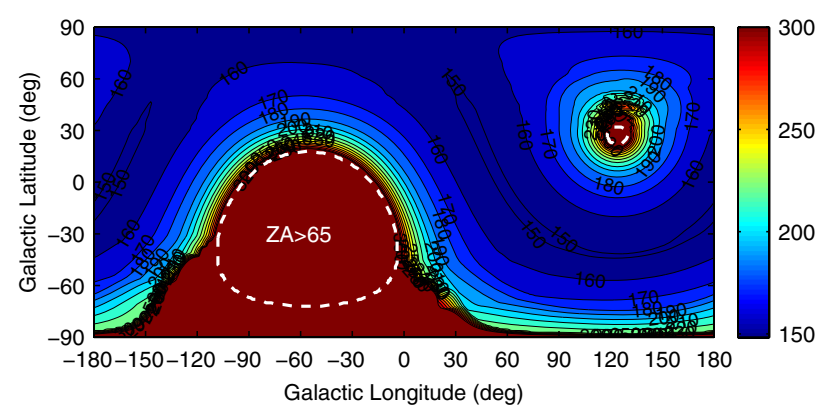

(a) $25 \%$ seeing

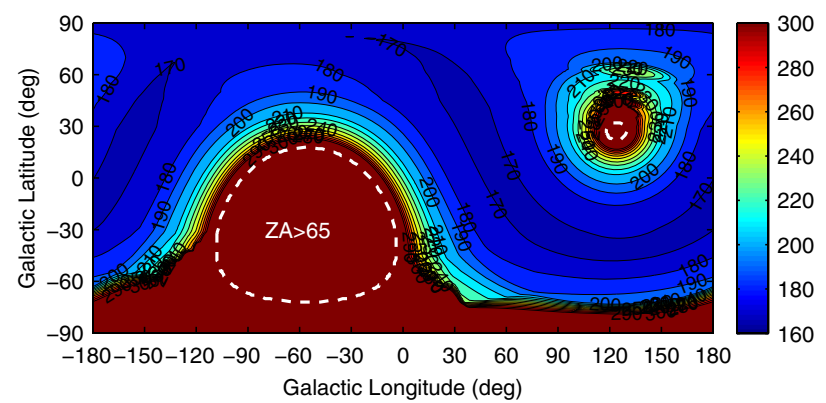

(b) median seeing

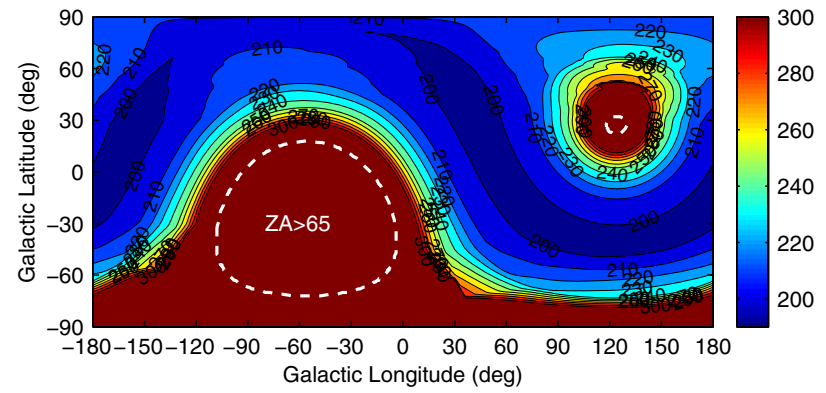

(c) $75 \%$ seeing

Fig. 8. (Color online) Map of total WFE at median sky coverage. Hour angle is $0 \mathrm{~h}$. 
zenith angle or $J \leq 19$ star counts in Fig. 5 . We observed a steep rise in the median sky coverage NGS mode WFE at high zenith angles and/or lower star counts.

These results can then be mapped onto galactic coordinates using interpolation based on the zenith angle and star density map shown in Figs. 1 and 3 . Figure 6 shows the results. We can see that the rms WFE in NGS modes at median sky coverage is less than $72 \mathrm{~nm}$ ( 2 mas) for the majority of the sky, except where the zenith angles are high $\left(>45^{\circ}\right)$.

The WFEs in NGS-controlled modes need to be combined with the WFE in LGS-controlled modes and implementation errors (optomechanics aberrations, etc.) to get the total WFE. We obtained the WFE in LGS-controlled modes by running simulations using MAOS with ideal NGS asterism (3 NGS on a small equilateral triangle) and project out NGS modes in the wavefront before computing WFE. Figure 7 shows the results.

The total WFE map is shown in Figs. $\underline{8}$ and 9 for hour angle of $0 \mathrm{~h}$ and $2 \mathrm{~h}$. The latter corresponds to the worst case performance during an integration

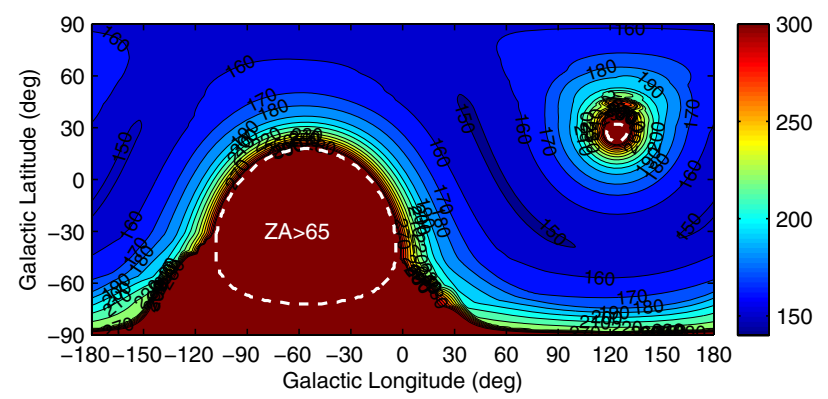

(a) $25 \%$ seeing

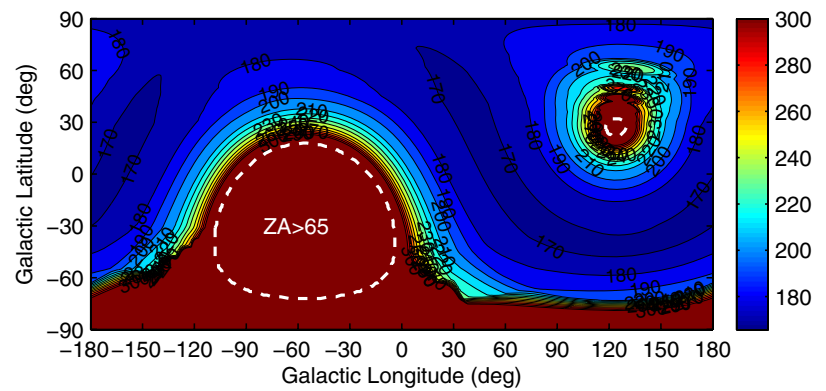

(b) median seeing

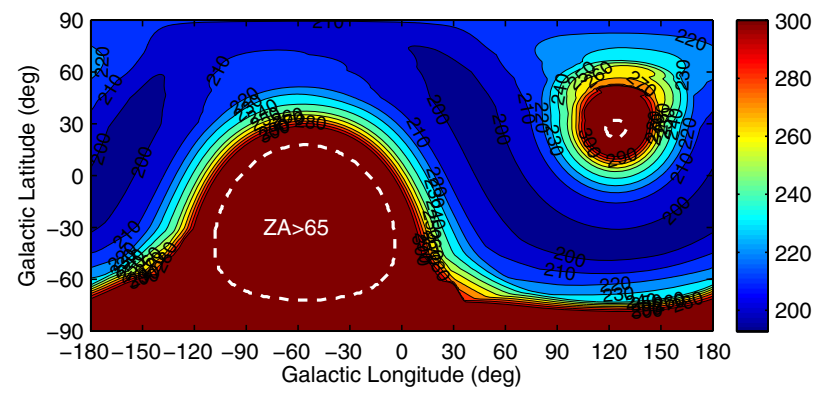

(c) $75 \%$ seeing

Fig. 9. (Color online) Map of total WFE at median sky coverage. Hour angle is $2 \mathrm{~h}$. time of $4 \mathrm{~h}$. These WFEs can be converted to Strehl ratios using the Marechal approximation. The $J$ band Strehl map is shown in Fig. 10. The best achievable $J$ band Strehls for median sky coverage are about $0.56,0.50$, and 0.38 for $25 \%$, median, and $75 \%$ seeing conditions within galactic latitudes of about $\pm 45^{\circ}$ with modest zenith angles. The $J$ band Strehl at the north galactic pole at median sky coverage is about $0.52,0.44$, and 0.32 for $25 \%$, median, and $75 \%$ seeing conditions.

Finally we computed the probability of meeting the requirement of $191 \mathrm{~nm}$ (230 nm for $75 \%$ seeing) total rms WFE ( $\mathrm{J}$ band Strehl is 0.4). The results are shown in Fig. 11. The $191 \mathrm{~nm}$ allowable WFE can be achieved more than $90 \%$ of the time at galactic latitudes less than $45^{\circ}$ for $25 \%$ and median seeing conditions for modest zenith angles $\left(\leq 45^{\circ}\right)$. A relaxed $230 \mathrm{~nm}$ WFE can be achieved for $75 \%$ seeing similarly. The sky coverage at the north galactic pole is about $85 \%$ and $75 \%$ for $25 \%$, median seeing conditions and $191 \mathrm{~nm}$ allowable WFE, and $70 \%$ for $75 \%$ seeing and $230 \mathrm{~nm}$ allowable WFE.

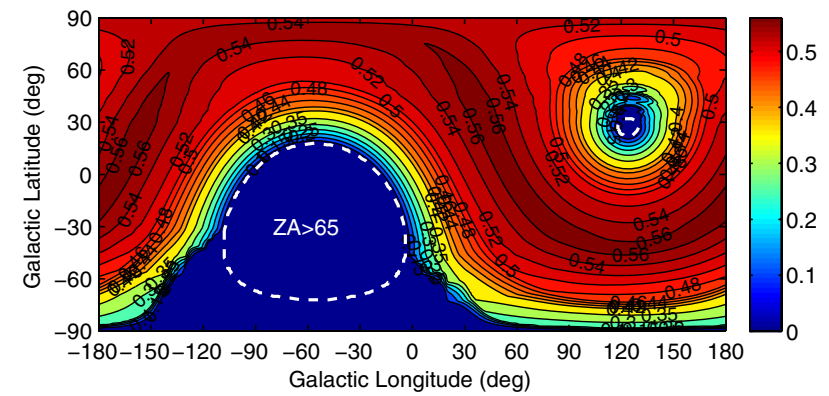

(a) $25 \%$ seeing

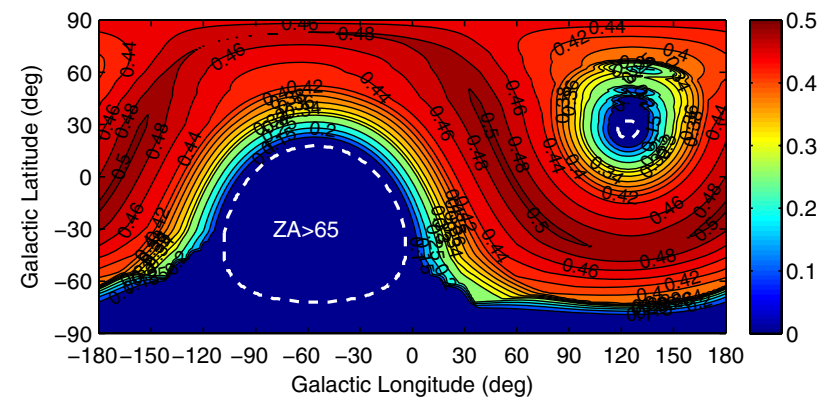

(b) median seeing

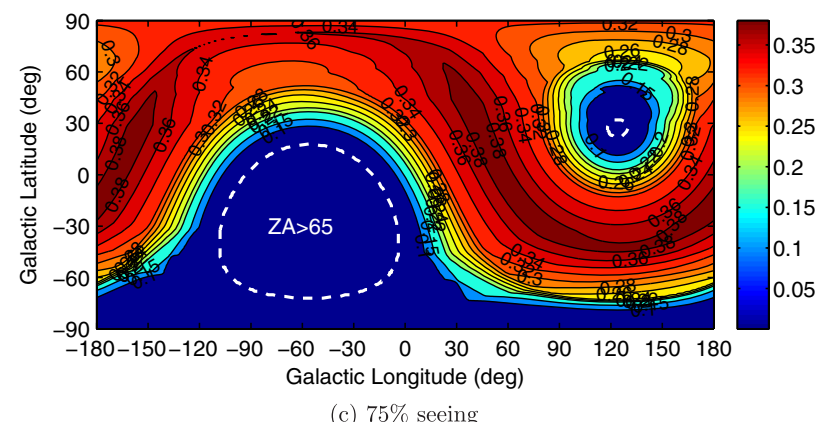

Fig. 10. (Color online) Map of $J$ band Strehl ratio map at median sky coverage. Hour angle is $0 \mathrm{~h}$. 


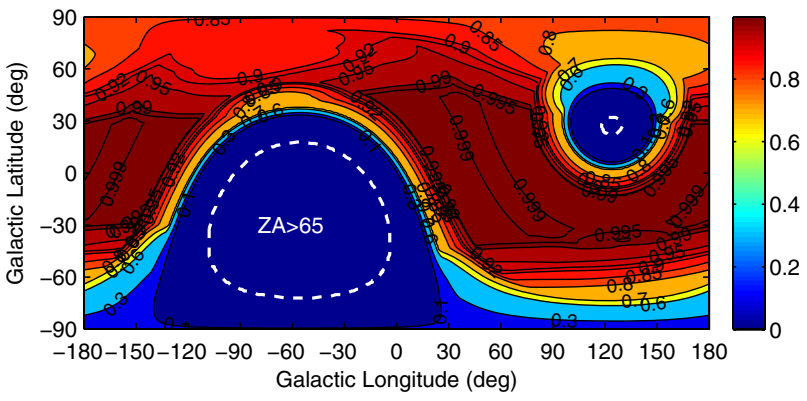

(a) $25 \%$ seeing

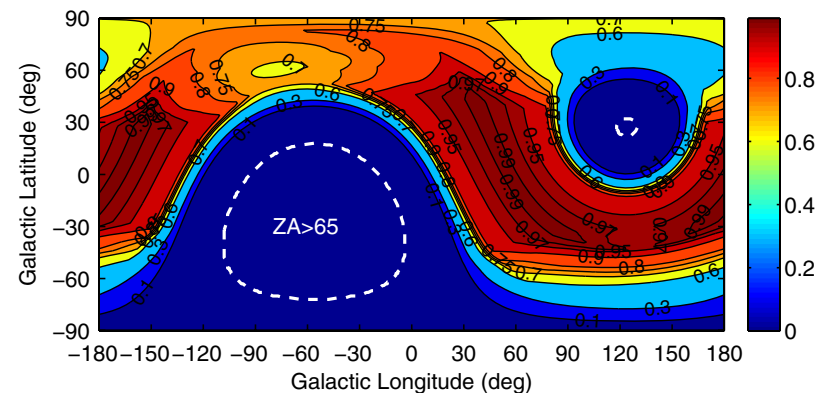

(b) median seeing

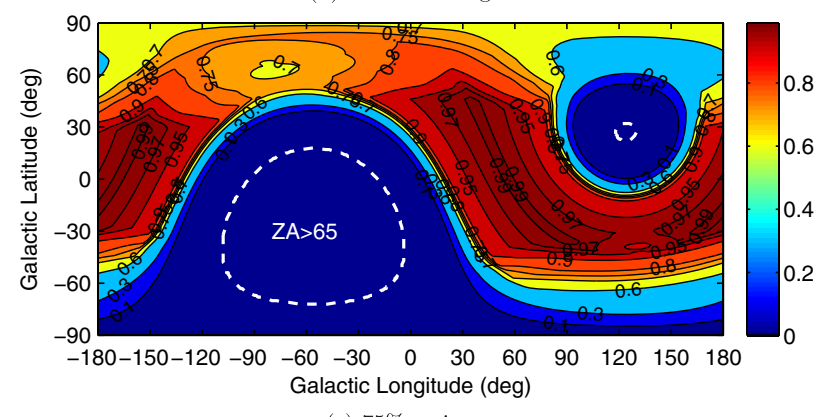

(c) $75 \%$ seeing

Fig. 11. (Color online) The probability to achieve $\leq 191 \mathrm{~nm}$ (or $230 \mathrm{~nm}$ for $75 \%$ seeing) total $\mathrm{rms}$ WFE.

\section{Conclusions}

In this paper, we presented the high fidelity sky coverage simulation results as a function of the galactic latitude for 0 and $2 \mathrm{~h}$ angle observations. This provides a direct view of the sky coverage for any given galactic latitude and longitude and should help astronomers to understand the potential of the $\mathrm{AO}$ system and plan the observations accordingly. In particular,

1. For median seeing and zero hour angle, NFIRAOS will deliver a WFE less than $191 \mathrm{~nm}$ more than $70 \%$ of the time over most of the observable sky.

2. The total WFE is less than 180 or $200 \mathrm{~nm}$ for an hour angle of 0 or $2 \mathrm{~h}$ over most of the observable sky for a typical asterism in either $25 \%$ or median seeing conditions.

The same technique can also be applied to evaluate sky coverage for a larger range of observing modes or conditions:

1. Spectrometer slit throughput

2. Observations with only a single TTF NGS

3. Long observations around an hour angle of 0 .
This work is supported by the TMT project. The authors gratefully acknowledges the support of the TMT partner institutions. They are the Association of Canadian Universities for Research in Astronomy (ACURA), the California Institute of Technology, and the University of California. This work was supported as well by the Gordon and Betty Moore Foundation, the Canada Foundation for Innovation, the Ontario Ministry of Research and Innovation, the National Research Council of Canada, the Natural Sciences and Engineering Research Council of Canada, the British Columbia Knowledge Development Fund, the Association of Universities for Research in Astronomy (AURA), and the U.S. National Science Foundation.

\section{References}

1. L. A. Thompson and R. M. Castle, "Experimental demonstration of a Rayleigh-scattered laser guide star at $351 \mathrm{~nm}$," Opt. Lett. 17, 1485-1487 (1992)

2. P. L. Wizinowich, D. Le Mignant, A. H. Bouchez, R. D. Campbell, J. C. Y. Chin, A. R. Contos, M. A. van Dam, S. K. Hartman, E. M. Johansson, R. E. Lafon, H. Lewis, P. J. Stomski, D. M. Summers, C. G. Brown, P. M. Danforth, C. E. Max, and D. M. Pennington, "The W. M. Keck Observatory laser guide star adaptive optics system: Overview," Publ. Astron. Soc. Pac. 118, 297-309 (2006).

3. M. Le Louarn, N. N. Hubin, and R. Arsenault, "Adaptive optics for second-generation VLT instruments," D. Bonaccini Calia, B L. Ellerbroek, and R. Ragazzoni, eds., Proc. SPIE, 5490, 248-259 (2004).

4. B. Neichel, F. Rigaut, M. Bec, M. Boccas, F. Daruich, C. D’Orgeville, V. Fesquet, R. Galvez, A. Garcia-Rissmann, G. Gausachs, M. Lombini, G. Perez, G. Trancho, V. Upadhya, and T. Vucina, "The Gemini MCAO System GeMS: nearing the end of a lab-story," Proc. SPIE, 7736, 773606 (2010).

5. B. Ellerbroek, S. Adkins, D. Andersen, J. Atwood, S. Browne, C. Boyer, P. Byrnes, K. Caputa, R. Conan, R. Cousty, D. Erikson, J. Fitzsimmons, F. Gamache, L. Gilles, G. Herriot, P. Hickson, O. Lardier, P. Morin, J. Pazder, T. Pfrommer, D. Quinn, V. Reshetov, S. Roberts, J.-C. Sinquin, M. Schoeck, M. Smith, G. Tyler, J. Vaughn, J.-P. Veran, C. Vogel, L. Wang, and I. Wevers, "First light adaptive optics systems and components for the Thirty Meter Telescope," Proc. SPIE, 7736, 773604 (2010).

6. E. Diolaiti, J.-M. Conan, I. Foppiani, M. Lombini, C. Petit, C. Robert, L. Schreiber, P. Ciliegi, E. Marchetti, M. Bellazzini, L. Busoni, S. Esposito, T. Fusco, N. Hubin, F. Quiros-Pacheco, A. Baruffolo, S. D'Odorico, J. Farinato, B. Neichel, R. Ragazzoni, C. Arcidiacono, V. Biliotti, G. Bregoli, G. Cosentino, and G. Innocenti, "A preliminary overview of the multiconjugate adaptive optics module for the E-ELT," Proc. SPIE 7015 , $7015 \mathrm{OU}$ (2008)

7. B. L. Ellerbroek and F. Rigaut, "Methods for correcting tilt anisoplanatism in laser-guide-star-based multiconjugate adaptive optics," J. Opt. Soc. Am. A 18, 2539-2547 (2001).

8. R. C. Flicker, F. J. Rigaut, and B. L. Ellerbroek, "Tilt anisoplanatism in laser-guide-star-based multiconjugate adaptive optics. Reconstruction of the long exposure point spread function from control loop data," Astron. Astrophys. 400, 1199-1207 (2003).

9. T. Fusco, A. Blanc, M. Nicolle, J.-L. Beuzit, V. Michau, G. Rousset, and N. Hubin, "Sky coverage estimation for multiconjugate adaptive optics systems: strategies and results," Mon Not. R. Astron. Soc. 370, 174-184 (2006).

10. R. M. Clare and B. L. Ellerbroek, "Sky coverage estimates for adaptive optics systems from computations in Zernike space," J. Opt. Soc. Am. A 23, 418-426 (2006). 
11. R. M. Clare, B. L. Ellerbroek, G. Herriot, and J.-P. Véran, "Adaptive optics sky coverage modeling for extremely large telescopes," Appl. Opt. 45, 8964-8978 (2006).

12. L. Wang, B. Ellerbroek, and J. P. Veran, "High fidelity sky coverage analysis via time domain adaptive optics simulations," Appl. Opt. 48, 5076-5087 (2009).

13. L. Wang, L. Gilles, and B. Ellerbroek, "Analysis of the improvement in sky coverage for multiconjugate adaptive optics systems obtained using minimum variance split tomography," Appl. Opt. 50, 3000-3010 (2011).

14. C. T. Jean-Pierre Veran, "Adaptive optics with Altair," Gemini Focus 33, 45-47 (2006).

15. S. V. W. Beckwith, M. Stiavelli, A. M. Koekemoer, J. A. R Caldwell, H. C. Ferguson, R. Hook, R. A. Lucas, L. E. Bergeron, M. Corbin, S. Jogee, N. Panagia, M. Robberto, P. Royle, R. S. Somerville, and M. Sosey, "The Hubble ultra deep field," Astron. J. 132, 1729-1755 (2006).

16. G. Herriot, D. Andersen, J. Atwood, C. Boyer, A. Beauvillier, P. Byrnes, R. Conan, B. Ellerbroek, J. Fitzsimmons, L. Gilles, P. Hickson, A. Hill, K. Jackson, O. Lardière, J. Pazder, T. Pfrommer, V. Reshetov, S. Roberts, J.-P. Véran, L. Wang, and I. Wevers, "NFIRAOS: TMT's facility adaptive optics system," Proc. SPIE, 7736, 77360B (2010).

17. M. S. Bessell and J. M. Brett, "JHKLM photometry—standard systems, passbands, and intrinsic colors," Publ. Astron. Soc. Pac. 100, 1134-1151 (1988).

18. A. Abahamid, J. Vernin, Z. Benkhaldoun, A. Jabiri, M. Azouit, and A. Agabi, "Seeing, outer scale of optical turbulence, and coherence outer scale at different astronomical sites using instruments on meteorological balloons," Astron. Astrophys. 422, 1123-1127 (2004).

19. B. L. Ellerbroek, "Efficient computation of minimum-variance wave-front reconstructors with sparse matrix techniques," J. Opt. Soc. Am. A 19, 1803-1816 (2002).

20. L. Gilles, L. Wang, and B. Ellerbroek, "Minimum variance split tomography for laser guide star adaptive optics," European J. Control 17, 327-334 (2011).
21. L. Wang, "Multithreaded adaptive optics simulator (maos)," http://lianqiw.github.com/maos/ (2011).

22. M. Schöck, S. Els, R. Riddle, W. Skidmore, T. Travouillon, R. Blum, E. Bustos, G. Chanan, S. G. Djorgovski, P. Gillett, B. Gregory, J. Nelson, A. Otárola, J. Seguel, J. Vasquez, A. Walker, D. Walker, and L. Wang, "Thirty Meter Telescope site testing I: overview," Publ. Astron. Soc. Pac. 121, 384-395(2009).

23. L. Gilles and B. Ellerbroek, "Shack-Hartmann wavefront sensing with elongated sodium laser beacons: centroiding versus matched filtering," Appl. Opt. 45, 6568-6576 (2006).

24. L. Gilles and B. L. Ellerbroek, "Constrained matched filtering for extended dynamic range and improved noise rejection for Shack-Hartmann wavefront sensing," Opt. Lett. 33, 1159-1161 (2008).

25. A. C. Robin, C. Reylé, S. Derrière, and S. Picaud, "A synthetic view on structure and evolution of the Milky Way," Astron. Astrophys. 409, 523-540 (2003).

26. http://model.obs-besancon.fr/.

27. J.-P. Veran and G. Herriot, "Type II woofer-tweeter control for NFIRAOS on TMT," in OSA Technical Digest (CD) (Optical Society of America, 2009), paper JTuC2.

28. D. G. MacMynowski and T. Andersen, "Wind buffeting of large telescopes," Appl. Opt. 49, 625-636 (2010).

29. G. Herriot, P. Hickson, B. Ellerbroek, J.-P. Véran, C.-Y. She, R. Clare, and D. Looze, "Focus errors from tracking sodium layer altitude variations with laser guide star adaptive optics for the Thirty Meter Telescope," Proc. SPIE, 6272, 62721 (2006).

30. M. M. James Binney, Galactic Astronomy (Princeton Univ., 1998)

31. S. G. Els, T. Travouillon, M. Schöck, R. Riddle, W. Skidmore, J. Seguel, E. Bustos, and D. Walker, "Thirty Meter Telescope site testing VI: turbulence profiles," Pub. Astron. Soc. Pac. 121, 527-543 (2009).

32. M. Schøck, mschoeck@tmt.org (personal communication, 2011). 